these measures was assessed using the intra-class correlation coefficient ( $\mathrm{Ri})$ and is expressed below as $\mathrm{Ri}(95 \% \mathrm{CI})$.

Results 145 subjects were recruited; 101 were male with a mean (SD) FEV1 (L) and FEV1/FVC ratio (\%) of $1.34 \mathrm{~L}(0.57)$ and 53\% (14.6) respectively.

Spirometry values showed excellent repeatability; FEV1 [0.93 (0.84 to 0.92$)$ and 0.89 (0.83 to 0.92$)]$ and FVC [0.80 (0.73 to 0.86$)$ and 0.81 (0.72 to 0.87 ) ] after 3 and 6 months respectively.

Sputum biomarkers of inflammation showed moderate repeatability at 3 and 6 months respectively; sputum neutrophils (\%) [0.59 (0.43 to 0.71$)$ and 0.50 (0.33 to 0.64$)]$ and eosinophils (\%) [0.62 ( 0.48 to 0.73 ) and 0.32 ( 0.13 to 0.49$)$ ].

The blood biomarkers peripheral blood white cell count (WCC), neutrophil and eosinophil counts demonstrated good repeatability after 3 and 6 month intervals respectively; WCC [0.68 (0.56 to 0.77) and 0.73 (0.62 to 0.81$)$ ], neutrophil count [0.66 (0.54 to 0.76$)$ and $0.71(0.59$ to 0.79$)]$ and eosinophil count $[0.66(0.54$ to 0.76$)$ and 0.73 (0.63 t o0.81)]. CRP showed fair repeatability [0.34 (0.16 to 0.5$)$ and 0.30 (0.11 to 0.47$)$ at both time intervals.

Discussions Sputum differential cell counts (\%) and peripheral blood differential cell counts are repeatable after 3 and 6 month intervals. These findings may have clinical implications when targeting therapies to sub-groups of COPD patients.

\section{P211 TIME-COURSE OF RHINOVIRUS AND BACTERIAL INFECTION DURING COPD EXACERBATION RECOVERY}

doi:10.1136/thoraxjnl-2012-202678.272

SN George, DS Garcha, ARC Patel, AJ Mackay, R Singh, RJ Sapsford, GC Donaldson, JA Wedzicha. University College London, London, United Kingdom

Introduction Airway bacteria and viruses are aetiological triggers of COPD exacerbations. Changes in prevalence of rhinovirus and typical airway bacteria together have not been examined during COPD exacerbation recovery. We compared the prevalence of the clinically relevant microorganisms (CRMs) human rhinovirus and typical pathogenic bacteria (H. influenzae, M. catarrhalis and S. pneumoniae) at five time points during COPD exacerbation recovery.

Methods London COPD cohort patients recorded any new or increased respiratory symptoms on daily diary cards and contacted the clinical team when symptoms worsened. Exacerbations were defined using our usual symptomatic criteria; an increase in respiratory symptoms for two consecutive days, with at least one symptom being major (dyspnoea, sputum purulence or volume) and the other a major or minor symptom (wheeze, cold, sore throat, cough). Reverse-transcription quantitative PCR was used to detect rhinovirus and real-time quantitative PCR was utilised to identify typical bacteria in sputum samples collected at exacerbation presentation (median 2 days after symptom onset), and at days 3, 7, 14 and 35 post-presentation.

Results Nineteen patients with moderate to severe COPD (mean age 68.8 years $(\mathrm{SD} \pm 8.1) ; \mathrm{FEV}_{1} 48.4 \%$ predicted $( \pm 19.2 \%$ ); current smoker 37\%; FEV ${ }_{1} / \mathrm{FVC} 0.46$ (SD \pm 0.14$)$; FEV $1.2 \mathrm{~L}(\mathrm{SD} \pm 0.4)$; male gender $74 \%$ ) provided 89 of 110 potential sputum samples at 5 time points during 22 exacerbations.

Rhinovirus prevalence progressively fell from $71.4 \%$ at exacerbation presentation to $0 \%$ at day 35 with significant decreases in prevalence between presentation and days 7, 14 and 35 (all $p<0.002$ ) (Figure 1). No exacerbation was negative for rhinovirus detection at presentation but positive at later time points. For typical bacteria, $64.7 \%$ of samples taken at presentation were positive. This proportion fell at days 3 and 7 but these falls were non-significant ( $p=0.08$ and $p=0.09$, respectively) - all events were treated with antibiotics. Seven of the 22 exacerbations (31.8\%) were positive for both CRMs at presentation.

Conclusion The prevalence of CRMs varies during recovery from a COPD exacerbation. Rhinovirus prevalence steadily decreases over 2 weeks whilst bacterial prevalence is more variable, presumably due to the background effects of lower airway bacterial colonisation. This emphasises the importance of rhinovirus as a major exacerbation trigger.

\section{P212 ASSESSING THE REPEATABILITY OF BACTERIAL DETECTION IN STABLE COPD USING SEVERAL METHODS}

doi:10.1136/thoraxjn-2012-202678.273

${ }^{1} \mathrm{BL}$ Barker, ${ }^{2} \mathrm{H}$ Patel, ${ }^{3} \mathrm{~K}$ Haldar, ${ }^{1} \mathrm{~V}$ Mistry, ${ }^{1} \mathrm{M}$ Pancholi, ${ }^{3} \mathrm{M}$ Barer, ${ }^{4} \mathrm{CE}$ Brightling, ${ }^{4} \mathrm{M}$ Bafadhel. 'Institute for Lung Health, University of Leicester, Leicester, United Kingdom; ${ }^{2}$ Department of Microbiology, University of Leicester NHS Hospitals, Leicester, United Kingdom; ' ${ }^{3}$ Department of Infection, Immunity and Inflammation, University of Leicester, Leicester, United Kingdom; "Institute for Lung Health, Department of Infection, Immunity and Inflammation, University of Leicester, Leicester, United Kingdom

Background Stable COPD patients are colonised if potentially pathogenic organisms are identified on sputum culture. Associations between colonisation and clinical features such as exacerbation

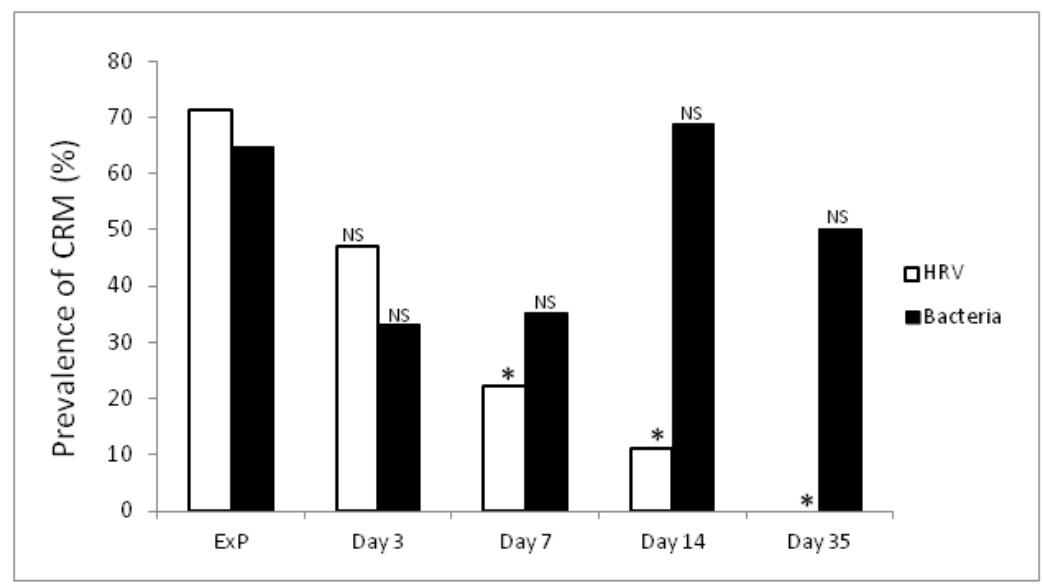

Figure 1. Changes in the prevalence of human rhinovirus (HRV) and typical bacteria at exacerbation and during recovery.

NS: No significant difference found between exacerbation present ation and each time point.

*: Significant decrease in prevalence between exacerbation presentation and each time point (all $\mathrm{p}<0.002$ )

Abstract P211 Figure 1 